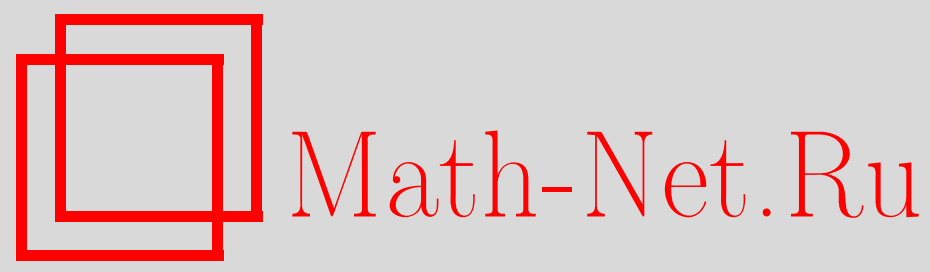

В. Т. Фоменко, Классификация двумерных поверхностей с нулевым нормальным кручением в четырехмерном пространстве постоянной кривизны, Матем. заметки, 2004, том 75 , выпуск $5,744-756$

DOI: https://doi.org/10.4213/mzm60

Использование Общероссийского математического портала Math-Net.Ru подразумевает, что вы прочитали и согласны с пользовательским соглашением http://www.mathnet.ru/rus/agreement

Параметры загрузки:

IP : 54.198 .55 .26

26 апреля 2023 г., 12:54:12

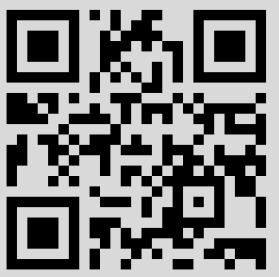




\title{
КЛАССИФИКАЦИЯ ДВУМЕРНЫХ ПОВЕРХНОСТЕЙ \\ С НУЛЕВЫМ НОРМАЛЬНЫМ КРУЧЕНИЕМ \\ В ЧЕТЫРЕХМЕРНОМ ПРОСТРАНСТВЕ ПОСТОЯННОЙ КРИВИЗНЫ
}

\section{В. Т. Фоменко}

\begin{abstract}
Вводится понятие нормального кручения поверхности в точке по заданному направлению в римановом пространстве. Дается полная классификация поверхностей в четырехмерном пространстве постоянной кривизны, для которых нормальное кручение тождественно равно нулю.

Библиограффия: 9 названий.
\end{abstract}

Впервые понятие нормального кручения в точке по заданному направлению для двумерной поверхности в четырехмерном евклидовом пространстве $E^{4}$ было введено В. Фесслером в 1959 г. в работе [1]. Затем в 1975 г. С.Б. Кадомцевьм [2] при некоторых дополнительных условиях было дано описание двумерных поверхностей с нулевым нормальным кручением в $E^{4}$. Эти исследования для поверхностей евклидова пространства были продолжены В. Т. Фоменко [3], [4], Л. А. Масальцевьм [5], Б. Ченом и Ш. Ли [6].

В данной работе вводится аналогичное понятие нормального кручения в точке по не асимптотическому направлению двумерной поверхности в римановом пространстве. Для четырехмерного риманова пространства постоянной кривизны $R^{4}$ дается полное описание двумерных поверхностей, не содержащих асимптотических направлений, с нулевым нормальным кручением для любой точки и по любому направлению поверхности.

1. Пусть $R^{4}$ - риманово пространство постоянной кривизны $R_{0}$, заданное метрической формой $d s^{2}=a_{\alpha \beta} d y^{\alpha} d y^{\beta}, a_{\alpha \beta} \in C^{k}, k \geqslant 4$. Рассмотрим в $R^{4}$ двумерную поверхность $F^{2}$, заданную в окрестности каждой своей точки уравнениями

$$
y^{\alpha}=f^{\alpha}\left(x^{1}, x^{2}\right), \quad\left(x^{1}, x^{2}\right) \in D, \quad \alpha=1, \ldots, 4,
$$

где $D$ - некоторая область параметрической плоскости $\left(x^{1}, x^{2}\right)$. Тензоры $t_{i}^{\alpha}=\partial y^{\alpha} / \partial x^{i}$ $\equiv \partial_{i} y^{\alpha}, i=1,2$, определяют касательную плоскость $T_{x} F^{2}$ поверхности $F^{2}$ в точке $x$. Метрика пространства $R^{4}$ индуцирует на $F^{2}$ метрику, задаваемую формулой $d s^{2}=$ $g_{i j} d x^{i} d x^{j}$, где $g_{i j}=a_{\alpha \beta} t_{i}^{\alpha} t_{j}^{\beta}$.

Работа выполнена при финансовой поддержке Российского фонда фундаментальных исследований, грант № 99-00814а. 
Рассмотрим регулярное нормальное оснащение поверхности $F^{2}$, задав его тензорными полями единичных взаимно-ортогональных векторов $n_{\sigma}^{\alpha}, \sigma=3,4$. Далее первые буквы греческого алфавита $\alpha, \beta, \gamma$. . будут пробегать значения от 1 до 4 ; латинские индексы $i, j, k$ - значения 1,2 ; греческие индексы $\sigma, \tau, \lambda$ - значения 3,4 . Будем также отождествлять одновалентные тензоры $t_{i}^{\alpha}, n_{\sigma}^{\alpha}, \ldots$ в точке $x$ в $R^{4}$ с векторами $t_{i}^{\alpha}, n_{\sigma}^{\alpha}, \ldots$, соприкасающимися к $R^{4}$ в точке $x$ евклидового пространства $E^{4}$. Все утверждения работы носят локальньй характер.

Введем в рассмотрение метрический тензор $g_{\sigma \tau}$ нормальной плоскости $N_{x} F^{2}$ поверхности $F^{2}$ в точке $x$, положив

$$
g_{\sigma \tau}=a_{\alpha \beta} n_{\sigma}^{\alpha} n_{\tau}^{\beta}
$$

Обозначим через $\bar{\nabla}=\stackrel{*}{\nabla} \oplus \nabla^{\perp}$ ковариантное дифференцирование в смысле Ван дер Вардена-Бортолотти, где $\stackrel{*}{\nabla}$ - смешанное ковариантное дифференцирование в $R^{4}$ вдоль $F^{2}$ (см., например, [7]), $\nabla^{\perp}-$ ковариантное дифференцирование в нормальной связности $F^{2}$.

Пусть $l_{g}: x^{i}=x^{i}(s)$ - геодезическая на поверхности $F^{2}$ в $R^{4}$, отнесенная к натуральному параметру. Пусть в каждой точке кривой $l_{g}$ однозначно определен единичный вектор первой нормали $m^{\alpha}$ в $R^{4}$. Рассмотрим ковариантную производную вектора $m^{\alpha}$ вдоль кривой $l_{g}: \bar{D} m^{\alpha} / d s$.

Пусть $\chi_{n}^{\alpha}-$ составляющая вектора $\bar{D} m^{\alpha} / d s$ в нормальной плоскости $N_{x} F^{2}$ поверхности $F^{2}$ в точке $x$. Длину вектора $\chi_{n}^{\alpha}$ будем назьвать нормальным. кручением поверхности $F^{2}$ в точке $x$ по направлению $t^{i}=d x^{i} / d s$ и обозначать через $\chi_{n}\left(x, t^{i}\right)$. Для поверхностей $F^{2}$ в $E^{4}$ величина $\chi_{n}\left(x, t^{i}\right)$ совпадает с кручением нормального сечения поверхности $F^{2}$ в точке $x$ по направлению $t^{i}$ (см. [3]).

В настоящей работе доказываются следующие теоремы.

Теорема 1. Пусть поверхность $F^{2}$ класса $C^{k}, k \geqslant 3$, в пространстве $R^{4}$ постоянной кривизны $R_{0}$ имеет плоскую нормальную связность и не имеет асимптотических направлений. Тогда нормальное кручение поверхности $F^{2}$ тождественно равно нулю $\left(\chi_{n}\left(x, t^{i}\right) \equiv 0 \quad \forall x \in F^{2}, \forall\left(t^{1}, t^{2}\right) \in T_{x} F^{2}\right)$ тогда и только тогда, когда поверхность $F^{2}$ удовлетворяет одному из следующих условий:

1) $F^{2}$ лежит на вполне геодезической гиперповерхности в $R^{4}$;

2) $F^{2}$ лехит на гиперповерхности постоянной кривизны $R_{0}+c_{1}^{2}, c_{1}>0$, имеет постоянную нулевую внутреннюю кривизну $K_{i} \equiv 0$ и ненулевой вектор средней кривизны постоянной длины $H_{0}, H_{0}^{2}+R_{0}>0$; әллипс нормальной кривизны в каждой точке поверхности вырохсдется в отрезок прямой постоянной длины $2 \sqrt{H_{0}^{2}+R_{0}}$ и составляет с вектором средней кривизны постоянный ненулевой угол $\varphi_{0}, \varphi_{0} \neq 0$, при этом $c_{1}^{2}=H_{0}^{2} \sin ^{2} \varphi_{0}$.

ТЕорема 2. Пусть нормальная связность поверхности $F^{2}$ класса $C^{k}, k \geqslant 4$, в пространстве $R^{4}$ постоянной кривизны $R_{0}$ не является плоской и поверхность не имеет асимптотических направлений. Тогда нормальное кручение поверхности $F^{2}$ равно нулю в любой точке и по любому направлению $\left(\chi_{n}\left(x, t^{i}\right) \equiv 0 \forall x \in F^{2}\right.$, $\left.\forall\left(t^{1}, t^{2}\right) \in T_{x} F^{2}\right)$ тогда и только тогда, когда выполнены следующие условия:

1) $F^{2}$ лежсит в пространстве $R^{4}$ постоянной положсительной кривизны $R_{0}=$ $3 c_{0}^{2}>0, c_{0}=$ const; 
2) $F^{2}$ имеет постоянную положительную внутреннюю кривизну $K_{i}=c_{0}^{2}>0$;

3) әллипс нормальной кривизны кажсой точки поверхности является окружностью радиуса с с с иентром в этой точке.

2. Выведем уравнения, описьвающие поверхности $F^{2}$ в $R^{4}$ с условием $\chi_{n}\left(x, t^{i}\right) \equiv 0$ $\forall x \in F^{2}, \forall\left(t^{1}, t^{2}\right) \in T_{x} F^{2}$. С этой целью введем в рассмотрение вторые квадратичные формы поверхности $F^{2}$ относительно нормали $n_{\sigma}^{\alpha}: I I_{\sigma}=b_{\sigma i j} d x^{i} d x^{j}$, где

$$
b_{\sigma i j}=a_{\alpha \beta} \stackrel{*}{\nabla}_{j} t_{i}^{\alpha} \cdot n_{\sigma}^{\beta}, \quad \stackrel{*}{\nabla}_{j} t_{i}^{\alpha}=\partial_{j} t_{i}^{\alpha}-\Gamma_{i j}^{k} t_{k}^{\alpha}+\stackrel{*}{\Gamma}_{\beta \gamma}^{\alpha} t_{i}^{\beta} t_{j}^{\gamma} ;
$$

$\Gamma_{i j}^{k}, \stackrel{*}{\Gamma}_{\beta \gamma}^{\alpha}-$ символы Кристоффеля, вычисленные, соответственно, по тензорам $g_{i j}$ и $a_{\alpha \beta}$.

Запишем уравнение геодезической $l_{g}: x^{i}=x^{i}(s)$ в пространственных координатах $y^{\alpha}$. Имеем $l_{g}: y^{\alpha}=f^{\alpha}\left(x^{1}(s), x^{2}(s)\right) \equiv F^{\alpha}(s)$. Используя формулы

$$
\frac{\bar{D} F^{\alpha}}{d s}=T^{\alpha}, \quad \frac{\bar{D} T^{\alpha}}{d s}=k m^{\alpha},
$$

где $k$ - кривизна кривой $l_{g}$ в $R^{4}$. Далее считаем, что $k \neq 0$. В силу формул Гаусса $\bar{\nabla}_{j} t_{i}^{\alpha}=b_{i j}^{\sigma} \cdot n_{\sigma}^{\alpha}$, где $b_{i j}^{\sigma}=g^{\sigma \tau} b_{\tau i j},\left\|g^{\sigma \tau}\right\|=\left\|g_{\sigma \tau}\right\|^{-1}$, имеем

$$
\frac{\bar{D} T^{\alpha}}{d s}=b_{i j}^{\sigma} \frac{d x^{i}}{d s} \frac{d x^{j}}{d s} \cdot n_{\sigma}^{\alpha} .
$$

Полагая $k^{\sigma}=b_{i j}^{\sigma} \frac{d x^{i}}{d s} \frac{d x^{j}}{d s}$ и учитывая, что $k^{2}=g_{\sigma \tau} k^{\sigma} k^{\tau}$, находим $m^{\alpha}=\frac{k^{\sigma}}{k} n_{\sigma}^{\alpha}$. Выполняя ковариантное дифференцирование $\bar{D} / d s$ левой и правой части этой формулы вдоль кривой $l_{g}$, убеждаемся, что обращение в нуль нормальной составляющей вектора $\bar{D} m^{\alpha} / d s$ в точках кривой $l_{g}$ эквивалентно выполнению соотношения

$$
\frac{\bar{D} k^{3}}{d s} \cdot k^{4}-k^{3} \frac{\bar{D} k^{4}}{d s}=0 .
$$

Перепишем это соотношение в виде $\bar{\nabla}_{k} b_{i j}^{[3} \cdot b_{l m}^{4]} t^{i} t^{j} t^{k} t^{l} t^{m}=0$, где квадратные скобки означают альтернирование по индексам.

Из полученной формулы следует, что условие $\chi_{n}\left(x, t^{i}\right) \equiv 0 \forall x \in F^{2}, \forall\left(t^{1}, t^{2}\right) \in T_{x} F^{2}$, эквивалентно системе уравнений

$$
\begin{aligned}
& b_{i i}^{[3} \bar{\nabla}_{i} b_{i i}^{4]}=0, \\
& 2 b_{i j}^{[3} \bar{\nabla}_{i} b_{i i}^{4]}+3 b_{i i}^{[3} \bar{\nabla}_{i} b_{i j}^{4]}=0, \\
& 6 b_{i j}^{[3} \bar{\nabla}_{j} b_{i i}^{4]}+3 b_{i i}^{[3} \bar{\nabla}_{j} b_{i j}^{4]}+b_{j j}^{[3} \bar{\nabla}_{i} b_{i i}^{4]}=0 .
\end{aligned}
$$

Ниже мы исследуем разрешимость системы (1), присоединив к ней систему уравнений Кодащпи-Гаусса-Риччи, записанную для поверхности $F^{2}$ :

$$
\begin{aligned}
\bar{\nabla}_{[i} b_{j] k}^{0} & =0 \\
\sum_{\sigma=3}^{4}\left(b_{11}^{\sigma} b_{22}^{\sigma}-b_{12}^{\sigma} b_{12}^{\sigma}\right) & =R_{12,12}-R_{0} \cdot g \\
g^{h l}\left(b_{l 2}^{3} b_{h 1}^{4}-b_{l 1}^{3} b_{h 2}^{4}\right) & =R_{12,34},
\end{aligned}
$$

где $g=\operatorname{det}\left\|g_{i j}\right\| ; R_{12,12}, R_{12,34}$ - компоненты тензоров кривизны, вычисленные, соответственно, по коэффициентам касательной $\Gamma_{j k}^{i}$ и нормальной связности $\Gamma_{i \tau}^{\sigma}=g^{\sigma \lambda} \Gamma_{\lambda, i \tau}$; $\Gamma_{\lambda, i \tau}=a_{\alpha \beta} n_{\lambda}^{\alpha} \cdot \stackrel{*}{\nabla}_{i} n_{\tau}^{\beta}$. Далее формы $d s^{2}, I I_{0}, \bar{\omega}_{0 \tau}=\Gamma_{0, i \tau} d x^{i}$ будем называть основными формами поверхности $F^{2}$. 
3. Будем отыскивать положительно определенный симметрический второго порядка тензор $g_{i j}$, тензор $b_{i j}^{\sigma}$ порядка $(2 ; 1)$, симметрический по индексам $i, j$, и коэффициенты нормальной связности $\Gamma_{i \tau}^{\sigma}$, удовлетворяющие системе $(1),(2)$ в области $D$ изменения переменных $x^{1}, x^{2}$.

Так как указанная система записана в тензорной форме, мы перепишем ее в конкретной системе координат $\left(x^{i}\right)$ на поверхности $F^{2}$ для специальным образом подобранного нормального оснашения $\left\{n_{\sigma}^{\alpha}\right\}$. Такой подход в решении систем $(1),(2)$ позволит считать заранее известными некоторые компоненты искомых величин. Этой цели служит следующая

Лемма. На поверхности $F^{2}$ класса $C^{k}, k \geqslant 3$, без асимптотических направлений существует параметризащия $\left(x^{i}\right)$ и регулярное нормальное оснащение $\left\{n_{\sigma}^{\alpha}\right\}$ такие, что

1) $b_{12}^{3} \equiv b_{12}^{4} \equiv 0$, если каждая точка $x$ поверхности $F^{2}$ лежст вне эллипса кривизны в $N_{x} F^{2} ; b_{11}^{3} \cdot b_{22}^{3} \neq 0, b_{11}^{4} \cdot b_{22}^{4} \neq 0$;

2) $b_{12}^{3} \equiv b_{11}^{4} \equiv b_{22}^{4} \equiv 0$, если каждая точка $x$ поверхности $F^{2}$ лежит внутри әллипса кривизнь в $N_{x} F^{2} ; b_{11}^{3} \cdot b_{22}^{3} \neq 0, b_{12}^{4} \neq 0$, при этом $g_{i j} \in C^{k-2}$, $b_{i j}^{\sigma} \in C^{k-2}, \Gamma_{i \tau}^{\sigma} \in C^{k-3}$.

ДокАЗАтЕльство. Так как на поверхности $F^{2}$ отсутствуют асимптотические направления, то каждая точка $x$ поверхности $F^{2}$ лежит либо вне эллипса нормальной кривизны в $N_{x} F^{2}$, либо внутри него. Пусть все точки поверхности $F^{2}$ являются внешними по отношению к эллипсу кривизны. Тогда одну из вторых квадратичных форм $b_{i j}^{\sigma} d x^{i} d x^{j}$ можно считать положительно определенной. Таким образом, на поверхности определено два семейства векторных полей класса $C^{k-2}$ таких, что квадратичные формы $I I_{\sigma}$, отнесенные к этим векторам в каждой точке поверхности, будут иметь канонический вид. Приняв интегральные кривые этих полей в качестве новой координатной сети, получим $b_{12}^{3} \equiv 0, b_{12}^{4} \equiv 0$, причем $g_{i j} \in C^{k-2}, b_{i j}^{\sigma} \in C^{k-2}, \Gamma_{i \tau}^{\sigma} \in C^{k-3}, k \geqslant 3$. При сделанном допущении лемма доказана.

Пусть каждая точка поверхности $F^{2}$ является внутренней по отношению к эллипсу кривизны в этой точке. Будем считать, что направления координатных линий $x^{i}$ в каждой точке поверхности являются асимптотическими относительно нормали $n_{4}^{\alpha}$, т.е. выполнены соотношения $b_{11}^{4} \equiv b_{22}^{4} \equiv 0, b_{11}^{3} \cdot b_{22}^{3}<0$.

Если $b_{12}^{3} \equiv 0$, то лемма доказана. Пусть $b_{12}^{3} \neq 0$. Проведем репер $\left\{n_{\sigma}^{\alpha}\right\}$ на угол $\alpha$, $\alpha \neq 0$, в нормальной плоскости в положение $\left\{n_{\sigma^{\prime}}^{\alpha}\right\}$, задав поворот репера формулами

$$
n_{3^{\prime}}^{\alpha}=\cos \alpha n_{3}^{\alpha}+\sin \alpha n_{4}^{\alpha}, \quad n_{4^{\prime}}^{\alpha}=-\sin \alpha n_{3}^{\alpha}+\cos \alpha n_{4}^{\alpha}
$$

Коэффициенты $b_{i j}^{\sigma^{\prime}}$ в новом репере принимают вид

$$
b_{i j}^{3^{\prime}}=\cos \alpha b_{i j}^{3}+\sin \alpha b_{i j}^{4}, \quad b_{i j}^{4^{\prime}}=-\sin \alpha b_{i j}^{3}+\cos \alpha b_{i j}^{4} .
$$

Покажем, что можно выбрать угол $\alpha$ класса $C^{k-2}$ и поле направлений $\left\{m^{1}: m^{2}\right\}$ и $\left\{n^{1}: n^{2}\right\}$ класса $C^{k-2}$ таким образом, чтобы выполнялись условия

$$
b_{i j}^{4^{\prime}} m^{i} m^{j}=0, \quad b_{i j}^{4^{\prime}} n^{i} n^{j}=0, \quad b_{i j}^{3^{\prime}} m^{i} n^{j}=0 .
$$


Так как $\alpha \neq 0$, то $b_{11}^{4^{\prime}} \neq 0$. Из первых двух уравнений системы (4) находим

$$
\frac{m^{1}}{m^{2}}+\frac{n^{1}}{n^{2}}=-\frac{2 b_{12}^{4^{\prime}}}{b_{11}^{4^{\prime}}}, \quad \frac{m^{1}}{m^{2}} \cdot \frac{n^{1}}{n^{2}}=\frac{b_{22}^{4^{\prime}}}{b_{11}^{4^{\prime}}} .
$$

В силу (5) третье уравнение системы (4) принимает вид

$$
b_{11}^{3^{\prime}} \cdot b_{22}^{4^{\prime}}-2 b_{12}^{3^{\prime}} \cdot b_{12}^{4^{\prime}}+b_{22}^{3^{\prime}} \cdot b_{11}^{4^{\prime}}=0
$$

Используя формулы (3), перепишем это выражение в виде

$$
\sin 2 \alpha\left(b_{11}^{3} \cdot b_{22}^{3}-b_{12}^{3} \cdot b_{12}^{3}+b_{12}^{4} \cdot b_{12}^{4}\right)+2 \cos 2 \alpha b_{12}^{3} \cdot b_{12}^{4}=0 .
$$

Так как каждая точка поверхности $F^{2}$ является внутренней по отношению к эллипсу кривизны в этой точке, то $b_{12}^{4} \neq 0$. Выберем угол $\alpha$ по формуле

$$
\operatorname{ctg} 2 \alpha=-\frac{b_{11}^{3} b_{22}^{3}-b_{12}^{3} b_{12}^{3}+b_{12}^{4} b_{12}^{4}}{2 b_{12}^{3} b_{12}^{4}} .
$$

При таком выборе угла $\alpha$ в новом соотношении $\left\{n_{\sigma^{\prime}}^{\alpha}\right\}$ имеют место формулы (4), если направления $\left\{m^{1}: m^{2}\right\}$ и $\left\{n^{1}: n^{2}\right\}$ выбраны по формулам (5). Так как $b_{i j}^{\sigma} \in C^{k-2}$, $k \geqslant 3$, то $\alpha \in C^{k-2}$ и потому $b_{i j}^{\sigma^{\prime}} \in C^{k-2}, k \geqslant 3$. Перейдем от координат $\left(x^{i}\right)$ к координатам $\left(x^{i^{\prime}}\right)$ таким образом, чтобы в каждой точке поверхности координатные линии $x^{i^{\prime}}$ имели выбранные направления $\left\{m^{1}: m^{2}\right\}$ и $\left\{n^{1}: n^{2}\right\}$. Тогда в новых координатах $\left(x^{i^{\prime}}\right)$ будем иметь $b_{1^{\prime} 1^{\prime}}^{4^{\prime}} \equiv b_{2^{\prime} 2^{\prime}}^{4^{\prime}} \equiv 0, b_{1^{\prime} 2^{\prime}}^{3} \equiv 0, g_{i^{\prime} j^{\prime}}, b_{i^{\prime} j^{\prime}}^{\sigma^{\prime}} \in C^{k-2}, k \geqslant 3, \Gamma_{i^{\prime} \tau^{\prime}}^{\sigma^{\prime}} \in C^{k-3}, k \geqslant 3$. Лемма доказана.

4. ДокаЗАТЕЛЬСТво тЕОРЕмы 1. Так как нормальная связность поверхности плоская, то на поверхности существует сеть линий кривизны. Примем ее в качестве координатной сети. Тогда $g_{12} \equiv b_{12}^{3} \equiv b_{12}^{4} \equiv 0$ для любого нормального оснащения. Выберем нормальный репер $\left\{n_{\sigma}^{\alpha}\right\}$ на поверхности таким образом, чтобы $\Gamma_{i \tau}^{\sigma} \equiv 0$. Из первого и второго уравнений системы (1) находим $b_{11}^{4}=\alpha_{0} b_{11}^{3}, b_{22}^{4}=\beta_{0} b_{22}^{3}$, где $\alpha_{0}=$ const, $\beta_{0}=$ const. Из первого уравнения системы $(2)$ с учетом полученных соотношений находим

$$
\partial_{2} g_{11} \cdot b_{22}^{3}\left(\beta_{0}-\alpha_{0}\right)=0, \quad \partial_{1} g_{22} \cdot b_{11}^{3}\left(\beta_{0}-\alpha_{0}\right)=0 .
$$

Исследуем эти соотношения.

a) Пусть $b_{11}^{3} \equiv 0$ (или $\left.b_{22}^{3} \equiv 0\right)$. Тогда $b_{11}^{4} \equiv 0$ (или $\left.b_{22}^{4} \equiv 0\right)$, и потому каждая точка поверхности является конщом эллипса кривизны, вырожденного в отрезок. Так как каждая точка поверхности $F^{2}$ не должна принадлежать эллипсу кривизны в этой точке, этот случай невозможен.

б) Пусть $\alpha_{0}=\beta_{0}$. Тогда все точки поверхности являются аксиальными, причем угол наклона отрезка индикатрисы кривизны к орту $n_{3}^{\alpha}$ постоянен на всей поверхности. Так как на поверхности $F^{2}$ отсутствуют асимптотические направления, отсюда вытекает, что поверхность $F^{2}$ лежит на вполне геодезической гиперповерхности (см., например, [8, c. 113]).

в) Пусть $b_{11}^{3} \neq 0, b_{22}^{3} \neq 0, \alpha_{0} \neq \beta_{0}$. Тогда из уравнения (7) следует, что $g_{11}=g_{11}\left(u^{1}\right)$, $g_{22}=g_{22}\left(u^{2}\right)$. Переходя к новым координатам $(u, \nu)$, не меняющим координатной сети 
на поверхности, можно добиться, чтобы $g_{11} \equiv 1, g_{22} \equiv 1, g_{12} \equiv 0$. Тогда в новых координатах из второго уравнения системы (2) находим $b_{11}^{3} b_{22}^{3}\left(1+\alpha_{0} \beta_{0}\right)+R_{0}=0$. Если $R_{0}=0$, то $1+\alpha_{0} \beta_{0}=0$, что приводит к тору Клиффорда (см. [3]). Эта поверхность лежит на гиперсфере, имеет постоянную среднюю и нулевую внутреннюю кривизну и ее индикатриса кривизны просматривается из точки поверхности под углом $\pi / 2$. Если $R_{0} \neq 0$, то $1+\alpha_{0} \beta_{0} \neq 0$, и потому $b_{22}^{3}=-R_{0} /\left(b_{11}^{3}\left(1+\alpha_{0} \beta_{0}\right)\right)$.

Подставляя это значение $b_{22}^{3}$ в третью формулу системы (1), находим $b_{11}^{3} \equiv$ const, $b_{22}^{3} \equiv$ const. Но тогда имеем $b_{11}^{4} \equiv$ const, $b_{22}^{4} \equiv$ const. Поворотом репера $\left\{n_{\sigma}^{\alpha}\right\}$ на постоянньй угол $\psi_{0}=\operatorname{arctg}\left(\left(b_{22}^{4}-b_{11}^{4}\right) /\left(b_{22}^{3}-b_{11}^{3}\right)\right)$ в положение $\left\{n_{\sigma^{\prime}}^{\alpha}\right\}$ можно добиться того, чтобы вторые квадратичные формы поверхности $F^{2}$ приняли вид $I I_{3}=b_{1} d u^{2}+b_{2} d \nu^{2}$, $I I_{4}=c_{1}\left(d u^{2}+d \nu^{2}\right), c_{1}>0$, где $b_{1}, b_{2}, c_{1}$ - постоянные, связанные в силу уравнения Гаусса соотношением $b_{1} b_{2}+c_{1}^{2}+R_{0}=0$; при этом в новом репере $\left\{n_{\sigma^{\prime}}^{\alpha}\right\}$ имеем $\Gamma_{i \tau^{\prime}}^{\sigma^{\prime}} \equiv 0$. Так как $g_{11}=g_{22} \equiv 1, g_{12} \equiv 0$, внутренняя кривизна $K_{i}$ поверхности тождественно равна нулю. Из явного вида вторых квадратичных форм поверхности $F^{2}$ следует, что средняя кривизна $H$ поверхности $F^{2}$ постоянна и дается формулой

$$
H=H_{0} \equiv \frac{1}{2} \sqrt{\left(b_{1}+b_{2}\right)^{2}+4 c_{1}^{2}} .
$$

Вектор средней кривизны $H^{\alpha}=\left(b_{1}+b_{2}\right) n_{3^{\prime}}^{\alpha} / 2+c_{1} n_{4^{\prime}}^{\alpha}$ имеет постоянньй угол $\varphi_{0}=$ $\operatorname{arctg}\left(2 c_{1} /\left(b_{1}+b_{2}\right)\right)$ с носителем индикатрисы кривизны, параллельным вектору $n_{3^{\prime}}^{\alpha}$ в плоскости $N_{x} F^{2}$, при этом длина $l$ индикатрисы кривизны постоянна на поверхности и дается формулой $l=2 \sqrt{H_{0}^{2}+R_{0}}$. Отсюда следует, что на рассматриваемой поверхности средняя кривизна $H_{0}$ удовлетворяет условию $H_{0}^{2}>-R_{0}$. Отметим, что по любым заданным величинам $H_{0}$ с условием $H_{0}^{2}>-R_{0}$ и $\varphi_{0}, \varphi_{0} \neq 0$, поверхность $F^{2}$ с метрикой $d s^{2}=d u^{2}+d \nu^{2}$ восстанавливается в $R^{4}$ всегда и однозначно (с точностью до движения в $\left.R^{4}\right)$.

Покажем, что поверхность $F^{2}$ лежит в $R^{4}$ на гиперповерхности $R_{1}^{3}$ кривизны $\left(R_{0}+c_{1}^{2}\right)$ $=$ const $\mathrm{c}$ неопределенньми линиями кривизны. С этой целью рассмотрим изометрическоепогружение метрики $d s^{2}=d u^{2}+d \nu^{2}$ в виде поверхности $\widetilde{F}^{2}$ со второй квадратичной формой $I I_{3}=b_{1} d u^{2}+b_{2} d \nu^{2}, b_{1}=$ const, $b_{2}=$ const, в пространство $R_{1}^{3}$ постоянной кривизны $\left(R_{0}+c_{1}^{2}\right), c_{1}^{2}=H_{0}^{2} \sin ^{2} \varphi_{0}$.

Так как уравнения Кодации для форм $d s^{2}$ и $I I_{3}$ в $R_{1}^{3}$ выполняются тождественно, а уравнение Гаусса выполняется в силу условия $b_{1} \cdot b_{2}=-\left(R_{0}+c_{1}^{2}\right)$, указанное погружение форм $d s^{2}$ и $I I_{3}$ в виде поверхности $\widetilde{F}^{2}$ с единичным нормальным вектором $\nu^{A}$ $(A=1,2,3)$ в $R_{1}^{3}$ возможно. Будем рассматривать $\widetilde{F}^{2}$ как поверхность в $R^{4}$, выбрав в качестве нормального оснащения для $\widetilde{F}^{2}$ единичный нормальньй вектор $\nu_{3}^{\alpha}$, лежащий в $R_{1}^{3}$ и совпадающий с вектором $\nu^{A}$, и единичный вектор нормали $\nu_{4}^{\alpha}$ нормальным к $R_{1}^{3}$ вдоль $\widetilde{F}^{2}$. Вектор уклонения $h^{\alpha}(x)$ точек $x+d x=\left(x^{1}+d x^{1}, x^{2}+d x^{2}\right)$ поверхности $\widetilde{F}^{2}$ от касательной геодезической к $\widetilde{F}^{2}$ в точке $x=\left(x^{1}, x^{2}\right)$ поверхности в $R^{4} \mathrm{c}$ точностью до членов высшего порядка относительно $d x^{1}, d x^{2}$ определяется формулой $h^{\alpha}(x)=\frac{1}{2} \widetilde{I I}^{\sigma} \nu_{\sigma}^{\alpha}$, где $\widetilde{I I}^{\sigma}=g^{\sigma \tau} \widetilde{I I}_{\tau}-$ вторые квадратичные формы поверхности $\widetilde{F}^{2}$, вычисленные относительно нормалей $\nu_{\sigma}^{\alpha}$. Так как $\widetilde{F}^{2}$ лежит в $R_{1}^{3} \subset R^{4}$, то составляющая $\frac{1}{2} \cdot \widetilde{I I}^{3} \nu_{3}^{\alpha}$ вектора уклонения $h^{\alpha}(x)$ представляет собой уклонение точки $(x+d x)$ поверхности $\widetilde{F}^{2}$ от касательной геодезической поверхности к $\widetilde{F}^{2}$ в точке $x$ в $R_{1}^{3}$ с точностью до бесконечно малых более высокого порядка относительно $d x^{1}, d x^{2}$ (см., например, [9]), т.е. совпадает с вектором $\frac{1}{2} I I_{3} \nu^{A}(A=1,2,3)$ в $R_{1}^{3}$. Так как единичный 
нормальный вектор $\nu^{A}$ к $\widetilde{F}^{2}$ в $R_{1}^{3}$ совпадает с $\nu_{3}^{\alpha}$ в $R^{4}$, отсюда следует, что $\widetilde{I I}_{3} \equiv I I_{3}$. Покажем теперь, что вторая квадратичная форма $\widetilde{I I} 4$ поверхности $\widetilde{F}^{2}$ в $R^{4}$ относительно нормали $\nu_{1}^{\alpha}$ совпадает с формой $I I_{4}=c_{1}\left(d u^{2}+d \nu^{2}\right)$. Так как гиперповерхность в $R_{1}^{3}$, имеющая кривизну $\left(R_{0}+c_{1}^{2}\right)$, погружена в $R^{4}$ кривизны $R_{0}$, имеет место формула [9] $R_{0}+c_{1}^{2}=(\Omega / 3)^{2}+R_{0}$, где $\Omega$ - средняя кривизна $R_{1}^{3}$. Отсюда следует, что $\Omega / 3=c_{1}$. За счет выбора направления $\nu_{4}^{\alpha}$ можно добиться того, чтобы знак $\Omega$ совпадал со знаком константы $c_{1}$. Так как все точки поверхности $R_{1}^{3}$ в силу постоянства ее кривизны являются омбилическими, нормальная кривизна $R_{1}^{3}$ в любой точке по любому направлению совпадает с константой $c_{1}$. Так как $\widetilde{F}^{2} \in R_{1}^{3}$, это означает, что вторая форма $\widetilde{F}^{2}$ относительно нормали $\nu_{4}^{\alpha}$ имеет вид $\widetilde{I I}_{4}=c_{1}\left(d u^{2}+d \nu^{2}\right), c_{1}^{2}=H_{0}^{2} \sin ^{2} \varphi_{0}$.

Покажем теперь, что для $\widetilde{F}^{2}$ в $R^{4}$ имеем $\widetilde{\omega}_{34}=a_{\alpha \beta} \nu_{3}^{\alpha} \cdot \stackrel{\nabla}{ }_{i} \nu_{4}^{\beta} \cdot d u^{i} \equiv 0$. Для этого продифференцируем тензор $\nu_{4}^{\alpha}$ ковариантно по переменной $x^{i}$ в $R^{4}$. Дифференцирование предполагает смешение по линии $x^{i}$ на $\widetilde{F}^{2}$ в $R^{4}$, а следовательно, смещение по $R_{1}^{3}$ в $R^{4}$. Так как линии кривизны на $R_{1}^{3}$ неопределены, любое направление на $R_{1}^{3}$ является главным и потому имеют место формулы Родрига

$$
\stackrel{\sim}{\nabla}_{i} \nu_{4}^{\alpha}=-\frac{\Omega}{3} t_{i}^{\alpha}
$$

Это означает, что тензор $\stackrel{*}{\nabla}_{i} \nu_{4}^{\alpha}$ принадлежит касательной плоскости к $\widetilde{F}^{2}$. Но тогда находим $a_{\alpha \beta} \nu_{3}^{\alpha} \cdot \stackrel{\nabla}{i}_{i} \nu_{4}^{\beta}=0$, т.е. на $\widetilde{F}^{2}$ имеем $\widetilde{\Gamma}_{i \tau}^{\sigma} \equiv 0$, и потому $\widetilde{\omega}_{34} \equiv \Gamma_{3, i 4} d u^{i} \equiv 0$. Таким образом, основные формы поверхностей $\widetilde{F}^{2}$ и $F^{2}$ в $R^{4}$ совпадают и в силу известной теоремы дифференциальной геометрии [9] поверхности $\widetilde{F}^{2}$ и $F^{2}$ в $R^{4}$ отличаются на движение. В связи с этим можно считать, что $F^{2}$ принадлежит в $R^{4}$ некоторой гиперповерхности постоянной кривизны $\left(R_{0}+c_{1}^{2}\right)$. Теорема 1 доказана.

5. ДоКАЗАТЕЛЬСТво ТЕОРЕмЫ 2. Пусть каждая точка поверхности является внешней по отношению к эллипсу кривизны в этой точке. Тогда в силу леммы на поверхности существует сопряженная координатная сеть, для которой $b_{12}^{3} \equiv b_{12}^{4} \equiv 0$. Из первого уравнения системы (1) при $i=1,2$ в этом случае следует, что

$$
\Gamma_{14}^{3}=\partial_{1}\left(\operatorname{arctg} \frac{b_{11}^{4}}{b_{11}^{3}}\right), \quad \Gamma_{24}^{3}=\partial_{2}\left(\operatorname{arctg} \frac{b_{11}^{4}}{b_{11}^{3}}\right) .
$$

Эти условия означают, что нормальная связность поверхности является плоской, а это противоречит условию теоремы. Следовательно, при сделанных предположениях в $R^{4}$ не существует поверхности с $\chi_{n}\left(x, t^{i}\right) \equiv 0 \forall x \in F^{2}, \forall\left(t^{1}, t^{2}\right) \in T_{x} F^{2}$.

Пусть каждая точка поверхности является внутренней по отношению к эллипсу кривизны в этой точке. Каждую поверхность, обладающую свойством $\chi_{n}\left(x, t^{i}\right) \equiv 0$ $\forall x \in F^{2}, \forall\left(t^{1}, t^{2}\right) \in T_{x} F^{2}$, будем обозначать через $F_{*}^{2}$.

Покажем, что на поверхности $F_{*}^{2}$ всегда существуют координаты $\left(x^{i}\right)$ и нормальное оснашение $\left\{n_{\sigma}^{\alpha}\right\}$ такие, что

$$
b_{11}^{4}=b_{22}^{4}=b_{12}^{3}=0, \quad b_{12}^{4} \cdot b_{12}^{4}+b_{11}^{3} \cdot b_{22}^{3}=0 .
$$

В самом деле, в силу леммы можно считать, что $b_{11}^{4}=b_{22}^{4}=b_{12}^{3}=0$. Тогда из первого уравнения системы (1) при $i=1$ и $i=2$ находим

$$
\Gamma_{13}^{4} \cdot b_{11}^{3}=2 \Gamma_{11}^{2} \cdot b_{12}^{4}, \quad \Gamma_{23}^{4} \cdot b_{22}^{3}=2 \Gamma_{22}^{2} \cdot b_{12}^{4} .
$$


Система (1) при этом принимает вид

$$
\begin{array}{cc}
\bar{\nabla}_{i} b_{i i}^{4}=0, \quad i=1,2, & \\
2 b_{i j}^{4} \bar{\nabla}_{i} b_{i i}^{3}-3 b_{i i}^{3} \bar{\nabla}_{i} b_{i j}^{4}=0, \quad i, j=1,2, & i \neq j, \\
2 b_{i j}^{4} \bar{\nabla}_{i} b_{j j}^{3}-b_{j j}^{3} \bar{\nabla}_{i} b_{i j}^{4}=0, \quad i, j=1,2, & i \neq j .
\end{array}
$$

Обратимся к уравнению Гаусса (21), переписав его в виде

$$
b_{11}^{3} b_{22}^{3}-b_{12}^{4} b_{12}^{4}=\widetilde{K} \cdot g,
$$

где $\widetilde{K}=R_{12,12} / g-R_{0}$.

Дифференцируя соотношение (12) по переменной $x^{i}$, находим

$$
\partial_{i} b_{11}^{3} \cdot b_{22}^{3}+b_{11}^{3} \partial_{i} b_{22}^{3}-2 b_{12}^{4} \partial_{i} b_{12}^{4}=\partial_{i} \tilde{K} \cdot g+\widetilde{K} \partial_{i} g, \quad i=1,2
$$

Воспользуемся формулой Фосса-Вейля $\partial_{i} \ln \sqrt{g}=\Gamma_{i 1}^{1}+\Gamma_{i 2}^{2}, i=1,2$, выражением для $\widetilde{K}$ из формулы (12) и перепишем полученное соотношение в виде

$$
\begin{gathered}
\left(\partial_{i} b_{11}^{3}-2 \Gamma_{i 1}^{1} b_{11}^{3}\right) b_{22}^{3}+b_{11}^{3}\left(\partial_{i} b_{22}^{3}-2 \Gamma_{i 2}^{2} b_{22}^{3}\right)-2 b_{12}^{4}\left(\partial_{i} b_{12}^{4}-\Gamma_{i 1}^{1} b_{12}^{4}-\Gamma_{i 2}^{2} b_{12}^{4}\right)=\partial_{i} \widetilde{K} \cdot g \\
i=1,2 .
\end{gathered}
$$

Запишем это соотношение, используя символику ковариантного дифференцирования $\bar{\nabla}$. Так как координаты $x^{i}$ на поверхности $F_{*}^{2}$ и оснащение $\left\{n_{\sigma}^{\alpha}\right\}$ вдоль $F_{*}^{2}$ выбраны так, что $b_{11}^{4}=b_{22}^{4}=b_{12}^{3}=0$, имеют место формулы

$$
\begin{aligned}
& \bar{\nabla}_{i} b_{11}^{3} \equiv \partial_{i} b_{11}^{3}-\Gamma_{i 1}^{k} b_{k 1}^{3}-\Gamma_{i 1}^{k} b_{1 k}^{3}+\Gamma_{i \sigma}^{3} b_{11}^{\sigma}=\partial_{i} b_{11}^{3}-2 \Gamma_{i 1}^{1} b_{11}^{3}, \\
& \bar{\nabla}_{i} b_{22}^{3} \equiv \partial_{i} b_{22}^{3}-\Gamma_{i 2}^{k} b_{k 2}^{3}-\Gamma_{i 2}^{k} b_{2 k}^{3}+\Gamma_{i \sigma}^{3} b_{22}^{\sigma}=\partial_{i} b_{22}^{3}-2 \Gamma_{i 2}^{1} b_{22}^{3}, \\
& \bar{\nabla}_{i} b_{12}^{4} \equiv \partial_{i} b_{12}^{4}-\Gamma_{i 1}^{k} b_{k 2}^{3}-\Gamma_{i 2}^{k} b_{1 k}^{4}+\Gamma_{i \sigma}^{3} b_{22}^{\sigma}=\partial_{i} b_{12}^{4}-2 \Gamma_{i 1}^{1} b_{12}^{4}-\Gamma_{i 2}^{2} b_{12}^{4} .
\end{aligned}
$$

Поэтому полученное вьше соотношение можно записать в виде

$$
\bar{\nabla}_{i} b_{11}^{3} b_{22}^{3}+b_{11}^{3} \bar{\nabla}_{i} b_{22}^{3}-2 b_{12}^{4} \bar{\nabla}_{i} b_{12}^{4}=\partial_{i} \widetilde{K} \cdot g, \quad i=1,2 .
$$

Соотношение (13) можно переписать в виде

$$
2 b_{i j}^{4} \bar{\nabla}_{i} b_{i j}^{4}=b_{j j}^{3} \bar{\nabla}_{i} b_{i i}^{3}+b_{i i}^{3} \bar{\nabla}_{i} b_{j j}^{3}-\partial_{i} \widetilde{K} \cdot g, \quad i, j=1,2, \quad i \neq j .
$$

Так как $b_{i j}^{4} \neq 0$ при $i \neq j$, находя из последнего соотношения величину $\bar{\nabla}_{i} b_{i j}^{4}$ и подставляя ее в уравнение (10), получим

$$
\left(-4 b_{i j}^{4} b_{i j}^{4}+3 b_{i i}^{3} b_{j j}^{3}\right) \bar{\nabla}_{i} b_{i i}^{3}+3 b_{i i}^{3} b_{i i}^{3} \bar{\nabla}_{i} b_{j j}^{3}-3 b_{i i}^{3} g \partial_{i} \widetilde{K}=0, \quad i, j=1,2, \quad i \neq j
$$

С другой стороны, исключив из уравнений $(10),(11)$ величину $\bar{\nabla}_{i} b_{i j}^{4}$, получим

$$
b_{j j}^{3} \bar{\nabla}_{i} b_{i i}^{3}=3 b_{i i}^{3} \bar{\nabla}_{i} b_{i j}^{3}, \quad i, j=1,2, \quad i \neq j .
$$


Подставляя $\bar{\nabla}_{i} b_{i j}^{3}$ из формул (15) в формулу (14), получим

$$
4\left(-b_{i j}^{4} b_{i j}^{4}+b_{i i}^{3} b_{j j}^{3}\right) \bar{\nabla}_{i} b_{i i}^{3}-3 b_{i i}^{3} g \partial_{i} \widetilde{K}=0, \quad i, j=1,2, \quad i \neq j .
$$

Это соотношение перепишем, положив сначала $i=1, j=2$, а затем $i=2, j=1$. Имеем

$$
4\left(-b_{12}^{4} b_{12}^{4}+b_{11}^{3} b_{22}^{3}\right) \bar{\nabla}_{1} b_{11}^{3}=3 b_{11}^{3} g \partial_{1} \widetilde{K}, \quad 4\left(-b_{12}^{4} b_{12}^{4}+b_{11}^{3} b_{22}^{3}\right) \bar{\nabla}_{2} b_{22}^{3}=3 b_{22}^{3} g \partial_{2} \widetilde{K}
$$

В силу уравнения Гаусса (12) отсюда следует, что

$$
\bar{\nabla}_{i} b_{i i}^{3}=b_{i i}^{3} \partial_{i} \ln \sqrt[4]{|\widetilde{K}|^{3}}, \quad i=1,2
$$

Раскрывая в этом выражении формулу ковариантной производной $\bar{\nabla}_{i} b_{i i}^{3}=\partial_{i} b_{i i}^{3}-$ $2 \Gamma_{i i}^{k} b_{k i}^{3}+\Gamma_{i 4}^{3} b_{i i}^{4}$ и используя формулу Фосса-Вейля, перепишем с учетом $b_{i i}^{4}=0, b_{i j}^{3}=0$ при $i \neq j$ соотношение (16) в виде

$$
\partial_{1} \ln \frac{\left|b_{11}^{3}\right|}{g \sqrt[4]{|\widetilde{K}|^{3}}}=-2 \Gamma_{12}^{2}, \quad \partial_{2} \ln \frac{\left|b_{22}^{3}\right|}{g \sqrt[4]{|\widetilde{K}|^{3}}}=-2 \Gamma_{21}^{1}
$$

В силу уравнения Гаусса (12) имеем

$$
\frac{\left|b_{11}^{3}\right|}{\sqrt[4]{|\widetilde{K}|^{3}}}=\frac{\left|b_{11}^{3}\right| \sqrt[4]{|\widetilde{K}|}}{\left|b_{11}^{3} b_{22}^{3}-b_{12}^{4} b_{12}^{4}\right|},
$$

поэтому соотношения (17) принимают вид

$$
-2 \Gamma_{i j}^{j}=\partial_{i} \ln \frac{\left|b_{i i}^{3}\right| \sqrt[4]{|\tilde{K}|}}{\left|b_{11}^{3} b_{22}^{3}-b_{12}^{4} b_{12}^{4}\right|}, \quad i \neq j
$$

В формуле (18) в левой части индекс $j$ не является индексом суммирования.

Далее из формулы (15) в силу формул (16) следует

$$
\frac{\bar{\nabla}_{i} b_{j j}^{3}}{b_{j j}^{3}}=\partial_{i} \ln \sqrt[4]{|\widetilde{K}|}
$$

Раскрывая знак ковариантной производной $\bar{\nabla}_{i} b_{j j}^{3}=\partial_{i} b_{j j}^{3}-2 \Gamma_{i j}^{k} b_{k j}^{3}+\Gamma_{i 4}^{3} b_{j j}^{4}$ и учитывая, что $b_{j j}^{4}=0, b_{k j}^{3}=0$ при $k \neq j$, соотношение (19) преобразуем к виду

$$
\partial_{i} \ln \frac{\sqrt[4]{|\widetilde{K}|}}{\left|b_{j j}^{3}\right|}=-2 \Gamma_{i j}^{j} \quad(\text { по индексу } j \text { не суммировать! }), \quad i, j=1,2, \quad i \neq j .
$$

Сравнивая полученную формулу с формулой (18), находим

$$
\partial_{i} \ln \frac{\left|b_{i i}^{3}\right| \cdot\left|b_{j j}^{3}\right|}{\left|b_{11}^{3} b_{22}^{3}-b_{12}^{4} b_{12}^{4}\right|}=0, \quad i, j=1,2, \quad i \neq j
$$


Отсюда следует, что $\left|b_{11}^{3}\right| \cdot\left|b_{22}^{3}\right|=c\left|b_{11}^{3} b_{22}^{3}-b_{12}^{4} b_{12}^{4}\right|$, где $c=$ const, $c>0$.

Так как рассматриваемая точка $x$ поверхности $F_{*}^{2}$ является внутренней по отношению к эллипсу кривизны в нормальной плоскости, а точки пересечения носителя вектора $n_{3}^{\alpha}$ с эллипсом кривизны соответствуют координатным направлениям $\{1,0\}$ и $\{0,1\}$ на поверхности в точке $x$, то $b_{11}^{3} \cdot b_{22}^{3}<0$ и потому предыдущее соотношение можно переписать в виде $b_{11}^{3} \cdot b_{22}^{3}=c\left(b_{11}^{3} b_{22}^{3}-b_{12}^{4} b_{12}^{4}\right)$. Полагая $q=(c-1) / c, q=$ const, отсюда находим

$$
b_{12}^{4} \cdot b_{12}^{4}=q b_{11}^{3} b_{22}^{3}
$$

Покажем, что $q=-1$.

Обратимся к уравнениям Кодацши (22). Полагая $\sigma=3$ и учитьвая, что $b_{12}^{3}=0$, $b_{i i}^{4}=0, i=1,2$, запишем их в силу формул $(8),(20)$ в виде

$$
\partial_{2} b_{11}^{3}=-(1+2 q) \cdot b_{22}^{3} \Gamma_{11}^{2}+b_{11}^{3} \Gamma_{12}^{1}, \quad \partial_{1} b_{22}^{3}=-(1+2 q) \cdot b_{11}^{3} \Gamma_{22}^{1}+b_{22}^{3} \Gamma_{12}^{2} .
$$

Полагая в формуле (15) $i=1, j=2$, а затем $i=2, j=1$, перепишем (15) с учетом (20), (8) в виде

$$
\begin{aligned}
& b_{22}^{3} \partial_{1} b_{11}^{3}=\left(2 \Gamma_{11}^{1}-3 \Gamma_{12}^{2}\right) b_{11}^{3} b_{22}^{3}-3(1+2 q) \Gamma_{22}^{1} b_{11}^{3} b_{11}^{3} \\
& b_{11}^{3} \partial_{2} b_{22}^{3}=\left(2 \Gamma_{22}^{2}-3 \Gamma_{12}^{1}\right) b_{22}^{3} b_{11}^{3}-3(1+2 q) \Gamma_{11}^{2} b_{22}^{3} b_{22}^{3}
\end{aligned}
$$

Из уравнений Кодащци при $\sigma=4$ находим с учетом (8), (20)

$$
\begin{aligned}
& b_{22}^{3} \partial_{1} b_{12}^{4}=\left(\Gamma_{11}^{1}-\Gamma_{12}^{2}\right) b_{12}^{4} b_{22}^{3}-2 \Gamma_{22}^{1} b_{12}^{4} b_{11}^{3} \\
& b_{11}^{3} \partial_{2} b_{12}^{4}=\left(\Gamma_{22}^{2}-\Gamma_{12}^{1}\right) b_{12}^{4} b_{11}^{3}+2 \Gamma_{11}^{2} b_{12}^{4} b_{22}^{3}
\end{aligned}
$$

Дифференцируя соотношение $(20)$ по переменной $x^{i}$, получим

$$
2 b_{12}^{4} \partial_{i} b_{12}^{3}=q\left(\partial_{i} b_{11}^{3} b_{22}^{3}+2 b_{11}^{3} \partial_{i} b_{11}^{3}\right), \quad i=1,2 .
$$

Подставляя сюда формулы $(22),(23)$, находим при $i=1$

$$
\begin{aligned}
2 b_{12}^{4}\left(\Gamma_{11}^{1} b_{12}^{4}-\Gamma_{12}^{2} b_{12}^{4}+2 \Gamma_{22}^{1} b_{12}^{4} \frac{b_{11}^{3}}{b_{22}^{3}}\right)= & q b_{22}^{3}\left[\left(2 \Gamma_{11}^{1}-3 \Gamma_{12}^{2}\right) b_{11}^{3}-3(1+2 q) \Gamma_{22}^{1} \frac{b_{11}^{3} b_{11}^{3}}{b_{22}^{3}}\right] \\
& +q b_{11}^{3}\left[-(1+2 q) \Gamma_{22}^{1} b_{11}^{3}+\Gamma_{12}^{2} b_{22}^{3}\right] .
\end{aligned}
$$

Отсюда следует, что

$$
8 b_{11}^{3}(1+q) \Gamma_{22}^{1}=0 .
$$

Аналогично, при $i=2$ находим

$$
8 b_{22}^{3}(1+q) \Gamma_{11}^{2}=0
$$

Так как $\Gamma_{i 4}^{3}, i=1,2$, одновременно не равны нулю, $b_{11}^{3} \neq 0, b_{22}^{3} \neq 0$, из формул (8), $(24),(25)$ следует, что $q=-1$. Это доказывает второе соотношение (7). Геометрический смысл последней формулы в (7) заключается в том, что каждая точка $x$ поверхности $F_{*}^{2}$ является фокусом эллипса кривизны в $N_{x} F_{*}^{2}$. 
Повернем репер $\left\{n_{\sigma}^{\alpha}\right\}$, существование которого доказано по формулам (7), на некоторьй угол $\stackrel{*}{\alpha}$ в положение $\left\{n_{\sigma^{\prime}}^{\alpha}\right\}$. Пусть $\left\{m_{1}: n_{1}\right\}$ и $\left\{m_{2}: n_{2}\right\}$ - направления в точке $x$, асимптотические относительно нормали $n_{4^{\prime}}^{\alpha}$. Покажем, что эти направления сопряжены относительно нормали $n_{3^{\prime}}^{\alpha}$ для любого значения $\stackrel{*}{\alpha}$. Для этого запишем условие сопряженности относительно $n_{3^{\prime}}^{\alpha}$ :

$$
\cos \stackrel{*}{\alpha} \cdot b_{11}^{3} m_{1} m_{2}+\sin \stackrel{*}{\alpha} \cdot b_{12}^{3}\left(m_{1} n_{2}+m_{2} n_{1}\right)+\cos \stackrel{*}{\alpha} \cdot b_{22}^{3} n_{1} n_{2}=0
$$

Учитывая, что $\left\{m_{1}: n_{1}\right\}$ и $\left\{m_{2}: n_{2}\right\}$ связаны соотношением

$$
\frac{m_{1}}{n_{1}} \cdot \frac{m_{2}}{n_{2}}=\frac{b_{22}^{3}}{b_{11}^{3}}, \quad \frac{m_{1}}{n_{1}}+\frac{m_{2}}{n_{2}}=2 \operatorname{ctg} \stackrel{*}{\alpha} \cdot \frac{b_{12}^{4}}{b_{11}^{3}},
$$

последнее соотношение приведем к виду

$$
2 \cos \stackrel{*}{\alpha}\left(b_{11}^{3} b_{22}^{3}+b_{12}^{4} b_{12}^{4}\right)=0
$$

Это соотношение выполняется тождественно для любого значения $\stackrel{*}{\alpha}$ в силу формул (7). Это означает, что для поверхности $F_{*}^{2}$ асимптотические направления относительно нормали $n_{4^{\prime}}^{\alpha}$ всегда являются сопряженньги для нормали $n_{3^{\prime}}^{\alpha}$ в любом ортонормированном репере $\left\{n_{\sigma^{\prime}}^{\alpha}\right\}$.

В дальнейшем считаем, что репер $\left\{n_{\sigma}^{\alpha}\right\}$ и координаты $\left(x^{i}\right)$ выбраны на $F_{*}^{2}$ так, что $g_{12}=b_{12}^{3}=b_{11}^{4}=b_{22}^{4}=0$. Этого всегда можно добиться, выбрав носитель $n_{3^{\prime}}^{\alpha}$, проходящим через центр эллипса кривизны в $N_{x} F_{*}^{2}$.

Интегрируя при этих условиях уравнения Кодацци, находим

$$
\left(\frac{b_{22}^{3}}{g_{22}}\right)^{2}=\left(\frac{b_{12}^{4}}{\sqrt{g_{11} g_{22}}}\right)^{3} \widetilde{U}^{-8}, \quad\left(\frac{b_{11}^{3}}{g_{11}}\right)^{2}=\left(\frac{b_{12}^{4}}{\sqrt{g_{11} g_{22}}}\right)^{3} \widetilde{V}^{-8},
$$

где $\widetilde{U}, \widetilde{V}$-некоторые функции переменных $x^{1}, x^{2}$ соответственно. Полагая $b_{11}^{3} / g_{11}=a^{2}$, $b_{22}^{3} / g_{22}=-b^{2}$, находим с учетом соотношения (7), что $a=\widetilde{U^{3}} \widetilde{V}>0, b=\widetilde{U} V^{3}>0$.

Если $\widetilde{U} \equiv \widetilde{V}$, то $\widetilde{U}=c_{0}=$ const, $\widetilde{V}=c_{0}=$ const, и тогда основные формы поверхности $F_{*}^{2}$ принимают вид

$$
\begin{gathered}
d s^{2}=E\left(d u^{2}+d \nu^{2}\right), \quad I I_{3}=c_{0} E\left(d u^{2}-d \nu^{2}\right) \\
I I_{4}=2 c_{0} E d u d \nu, \quad \omega=-\frac{E_{\nu}}{E} d u+\frac{E_{u}}{E} d \nu .
\end{gathered}
$$

где $c_{0}=$ const, a функция $E$ находится из систем уравнений $(1),(2)$. Следует отметить, что все уравнения систем $(1),(2)$, кроме уравнений Гаусса и Риччи вьполняются тождественно. Уравнение Гаусса имеет вид $-\Delta \ln E=2 E\left(-2 c_{0}^{2}+R_{0}\right)$, где $R_{0}-$ кривизна пространства $R^{4}, \Delta$ - оператор Лапласа. Уравнение Риччи для форм (26) имеет вид $-\Delta \ln E=2 c_{0}^{2} E$.

Сравнивая уравнения Гаусса и Риччи, находим $R_{0}=3 c_{0}^{2}$, при этом $\left(-\frac{1}{2 E} \Delta \ln E\right)=c_{0}^{2}$. Это означает, что поверхность $F_{*}^{2}$ имеет постоянную внутреннюю кривизну $K_{i}=c_{0}^{2}>0$ и является аналогом куска поверхности Веронезе в $S^{4} \subset E^{5}$. 
Пусть $0<\widetilde{U}<\widetilde{V}$. Тогда в силу уравнений Кодащпи имеем

$$
g_{11}=\tilde{c}_{1}^{4}\left(x^{1}\right) \frac{\left|\widetilde{U}^{4}-\widetilde{V}^{4}\right|}{\widetilde{V}^{4}}, \quad g_{22}=\tilde{c}_{2}^{4}\left(x^{2}\right) \frac{\left|\widetilde{U}^{4}-\widetilde{V}^{4}\right|}{\widetilde{U}^{4}},
$$

где $\tilde{c}_{1}$ и $\tilde{c}_{2}-$ некоторые положительные функции своих аргументов. Полагая

$$
u=\int \widetilde{U}^{2}\left(x^{1}\right) \tilde{c}_{1}^{2}\left(x^{1}\right) d x^{1}, \quad \nu=\int \widetilde{V}^{2}\left(x^{2}\right) \tilde{c}_{2}^{2}\left(x^{2}\right) d x^{2},
$$

находим основные формы поверхности $F_{*}^{2}$ в виде

$$
\begin{gathered}
d s^{2}=\left(U^{2}-V^{2}\right)\left(d u^{2}+d \nu^{2}\right), \quad U>V>0, \\
I I_{3}=\frac{U^{2}-V^{2}}{U^{3} V} d u^{2}-\frac{U^{2}-V^{2}}{U V^{3}} d \nu^{2}, \quad I I_{4}=\frac{2\left(U^{2}-V^{2}\right)}{U^{2} V^{2}} d u d \nu, \\
\omega=-\frac{2 U V^{\prime}}{U^{2}-V^{2}} d u-\frac{2 V U^{\prime}}{U^{2}-V^{2}} d \nu
\end{gathered}
$$

где $U, V$ - дважды дифференцируемые функции аргументов $u, \nu$ соответственно.

Отметим, что коэффициенты форм (27) удовлетворяют уравнениям систем (1) и (2), кроме уравнений Гаусса и Риччи. Для нахождения функций $U, V$ вьпишем уравнения Гаусса и Риччи. Уравнение Гаусса имеет вид

$$
-\frac{1}{2 E} \Delta \ln E=\frac{b_{11}^{3} b_{22}^{3}-b_{12}^{4} b_{12}^{4}}{E^{2}} R_{0},
$$

где $E=U^{2}-V^{2}$. Выполняя дифференцирование функции $E$, находим

$$
U U^{\prime \prime}-V V^{\prime \prime}=\frac{U^{2}+V^{2}}{U^{2}-V^{2}}\left(U^{\prime 2}-V^{\prime}\right)+2 \frac{\left(U^{2}-V^{2}\right)^{3}}{U^{4} V^{4}}-R_{0}\left(U^{2}-V^{2}\right)^{2},
$$

где знак “ " ” означает дифференцирование.

Уравнение Риччи имеет вид

$$
\partial_{2} \Gamma_{14}^{3}-\partial_{1} \Gamma_{24}^{3}=\frac{1}{E}\left(b_{22}^{3} b_{12}^{4}-b_{11}^{3} b_{12}^{4}\right), \quad \text { где } E=U^{2}-V^{2} .
$$

Подставляя сюда значения $\Gamma_{i 4}^{3}, b_{i j}^{\sigma}$ из формул (27) и вьполняя дифференцирование, находим

$$
V U^{\prime \prime}-U V^{\prime \prime}=\frac{2 U V}{U^{2}-V^{2}}\left(U^{\prime 2}+V^{\prime 2}\right)-\frac{\left(U^{2}-V^{2}\right)\left(U^{2}+V^{2}\right)}{2 U^{5} V^{5}} .
$$

Из соотношений $(28),(29)$ получаем

$$
U^{\prime \prime}=\frac{U}{U^{2}-V^{2}}\left(U^{\prime 2}+V^{\prime 2}\right)+c_{1}, \quad V^{\prime \prime}=-\frac{V}{U^{2}-V^{2}}\left(U^{\prime 2}+V^{\prime 2}\right)+c_{2},
$$

где

$c_{1}=\frac{5 U^{4}-4 U^{2} V^{2}-V^{4}}{2 U^{5} V^{4}}-R_{0} U\left(U^{2}-V^{2}\right), \quad c_{2}=\frac{-5 V^{4}+4 U^{2} V^{2}+U^{4}}{2 U^{4} V^{5}}-R_{0} V\left(U^{2}-V^{2}\right)$.

Дифференцируя первое уравнение системы (30) по переменной $\nu$, а второе - по $u$, и подставляя в полученньй результат производные $\partial_{1} c_{2}, \partial_{2} c_{1}$, находим

$$
V^{\prime}\left(-9 V^{4}+18 U^{2} V^{2}-9 U^{4}\right)=0, \quad U^{\prime}\left(9 U^{4}-18 U^{2} V^{2}+9 V^{4}\right)=0 .
$$

Так как $U^{2}-V^{2}>0$, отсюда следует, что $U^{\prime}=V^{\prime}=0$. Но тогда из формул (15) следует, что $\omega \equiv 0$. Это означает, что нормальная связность поверхности $F_{*}^{2}$ является плоской. Полученное противоречие означает, что поверхностей типа $F_{*}^{2}$ с основными формами вида (27) в пространстве $R^{4}$ не существует. Теорема 2 доказана.

Автор признателен рецензенту за указанные замечания. 


\section{СПИСОК ЦИТИРОВАННОЙ ЛИТЕРАТУРЫ}

[1] Fessler W. Über die normaltorsion von Flachen im vierdimensionalen euklidischen Raum // Comm. Math. Helv. 1959. V. 33. №2. P. 89-108.

[2] Кадомцев С. Б. Исследование некоторых свойств нормального кручения 2-мерных поверхностей в 4-мерном пространстве // Итоги науки и техники. Проблемы геометрии. Т. 7. М.: ВИНИТИ, 1975. С. 267-278.

[3] Фоменко В. Т. Некоторые свойства двумерных поверхностей с нулевым нормальным кручением в $E^{4} / /$ Матем. сб. 1978. Т. 106 (148). № 4.

[4] Фоменко В. Т. Некоторые результаты теории двумерных поверхностей в $E^{4} / /$ Деп. ВИНИТИ № 4062-И87. М.: ВИНИТИ, 1987. С. 2-118.

[5] Масальцев Л. А. О двумерных поверхностях с нулевым нормальным кручением // УГС. 1984. T. 27. C. $88-91$.

[6] Bang-yen Chen, Shi-jie Li. Classification of surfaces with pointwise planar normal sections and its application to Fomenko's cojecture // J. Geometry. 1986. V. 26. P. 21-34.

[7] Рашевский П. К. Риманова геометрия и тензорный анализ. М.: Наука, 1964.

[8] Схоутен И. А., Стройк Д. Дж. Введение в новые методы дифференциальной геометрии. Т. II. М: ГИИЛ, 1948.

[9] Эйзенхарт Л. П. Риманова геометрия. М.: ГИИЛ, 1948.

Таганрогский государственный педагогический институт

Поступило 10.11.2001

Исправленный вариант

04.09 .2003 